\title{
A connection between quadrature formulas on the unit circle and the interval $[-1,1]$
}

\author{
Adhemar Bultheel ${ }^{\mathrm{a}, *}$ Leyla Daruis ${ }^{\mathrm{b}, * *}$ and Pablo González-Vera ${ }^{\mathrm{b}, * * *}$ \\ ${ }^{a}$ Department of Computer Science, K.U.Leuven, Belgium \\ E-mail: Adhemar.Bultheel@cs.kuleuven.ac.be \\ ${ }^{b}$ Department of Mathematical Analysis, University La Laguna, Tenerife, Spain \\ E-mail: pglez@ull.es
}

\begin{abstract}
We establish a relation between Gauss quadrature formulas on the interval $[-1,1]$ that approximate integrals of the form $I_{\sigma}(F)=\int_{-1}^{+1} F(x) \sigma(x) \mathrm{d} x$ and Szego quadrature formulas on the unit circle of the complex plane that approximate integrals of the form $\tilde{I}_{\omega}(f)=\int_{-\pi}^{\pi} f\left(e^{i \theta}\right) \omega(\theta) \mathrm{d} \theta$. The weight $\sigma(x)$ is positive on $[-1,1]$ while the weight $\omega(\theta)$ is positive on $[-\pi, \pi]$. It is shown that if $\omega(\theta)=\sigma(\cos \theta)|\sin \theta|$, then there is an intimate relation between the Gauss and Szegő quadrature formulas. Moreover, as a side result we also obtain an easy derivation for relations between orthogonal polynomials with respect to $\sigma(x)$ and orthogonal Szegő polynomials with respect to $\omega(\theta)$. Inclusion of Gauss-Lobatto and Gauss-Radau formulas is natural.

Keywords: numerical quadrature, Gauss quadrature, Szegő quadrature, Gauss-Lobatto formula, GaussRadau formula
\end{abstract}

AMS Subject classification: $41 \mathrm{~A} 55,42 \mathrm{C} 05$

\section{Introduction}

Gaussian quadrature formulas are well known. They are formulas of the form $I_{n}(F)=$ $\sum_{k=1}^{n} \lambda_{k} F\left(x_{k}\right)$. If they are used to approximate an integral $I_{\sigma}(F)=\int_{-1}^{+1} F(x) \sigma(x) \mathrm{d} x$, then the nodes $x_{k}$ are the zeros of the $n$-th polynomial with respect to the weight function $\sigma(x)$, i.e., orthogonal with respect to the inner product $\langle F, G\rangle=\int_{-1}^{+1} F(x) G(x) \sigma(x) \mathrm{d} x$. All these nodes are distinct and belong to the open interval $(-1,1)$. The coefficients are all positive. These formulas have a maximal degree of accuracy because $I_{n}(F)=I_{\sigma}(F)$ for all $F$ that are polynomials of degree at most $2 n-1$.

Szegö formulas are of the form $\tilde{I}_{n}(f)=\sum_{k=1}^{n} \tilde{A}_{k} f\left(z_{k}\right)$, and they approximate integrals of the form $\tilde{I}_{\omega}(f)=\int_{-\pi}^{\pi} f\left(e^{i \theta}\right) \omega(\theta) \mathrm{d} \theta$, where $\omega(\theta)$ is a positive weight function for the interval $[-\pi, \pi]$. The polynomials $\varphi_{n}(z)$ that are orthogonal with respect to the inner product $\langle f, g\rangle=\int_{-\pi}^{\pi} f\left(e^{i \theta}\right) \overline{g\left(e^{i \theta}\right)} \omega(\theta) \mathrm{d} \theta$ are known to have all their zeros in the open unit disk $\mathbb{D}$. So if we want the nodes $z_{k}$ to be on the unit circle, we have to take them as the zeros of the so called para-orthogonal polynomials $B_{n}(z)=\varphi_{n}(z)+\tau z^{n} \overline{\varphi_{n}(1 / \bar{z})}$, where $|\tau|=1$. The $n$ zeros of $B_{n}(z)$ are simple and lie on the unit circle. The corresponding weights $\tilde{A}_{k}$ are positive and these quadrature formulas have a maximal domain of validity, namely $\tilde{I}_{n}(f)=\tilde{I}_{\omega}(f)$ for all Laurent polynomials $f$ of the form $f(x)=x^{-n+1} P(x)$, with

\footnotetext{
* This work is partially supported by the Belgian Program on Interuniversity Poles of Attraction, initiated by the Belgian State, Prime Minister's Office for Science, Technology and Culture. The scientific responsibility rests with the authors.

** The work of this author was performed as part of a grant of the Gobierno de Canarias.

${ }^{* * *}$ The work of this author was supported by the Scientific Research Project of the Spanish D.G.E.S. under contract PB96-1029.
} 
$P(x)$ a polynomial of degree $2 n-2$ at most, i.e., a linear subspace of dimension $2 n-1$ of the space of polynomials.

A systematic approach to quadrature formulas on the unit circle was undertaken by Jones, Njåstad and Thron [6].

It will be shown in this paper that there is an intimate relationship between the Gauss and the Szegö quadrature formulas if $\sigma(x)$ and $\omega(\theta)$ are related by $\omega(\theta)=\sigma(\cos \theta)|\sin \theta|$. By relating the complex variable $z$ and the real variable $x$ through the variable $\theta$ as $z=e^{i \theta}$ and $x=\cos \theta$, one gets a direct relation between the nodes $x_{j}$ in $[-1,1]$ and the nodes $z_{j}$ on the unit circle. The symmetry of the weight $\omega(\theta)$ implies a symmetry (complex conjugate pairs or real) in the nodes $z_{k}$ if the parameter $\tau$ is chosen real, i.e., $\tau= \pm 1$. This allows us to relate $I_{n}$ and $\tilde{I}_{2 n}$ for $\tau=1$. On the other hand, because for $\tau=-1$, also $z= \pm 1$ are nodes of the Szegö formula $\tilde{I}_{2 n+2}$, this does not correspond to a Gauss formula, but to a $(n+2)$-point Gauss-Lobatto formula, which includes the end points $x= \pm 1$ as nodes of the quadrature formula.

For Szegö formulas having an odd index $\tilde{I}_{2 n+1}$, either $z=+1$ or $z=-1$ is a node, and the corresponding quadrature formula on the interval is a $(n+1)$-point Gauss-Radau formula, which includes one of the end points of the interval $[-1,1]$.

Because these nodes are zeros of orthogonal, respectively para-orthogonal polynomials, this also leads to a relation between these different polynomials. Of course the relation between polynomials orthogonal on an interval and polynomials orthogonal on the unit circle is known. See e.g. [8, Sec. 11.5], [4, Chap. IX], [5, Sec. 6.4$]$.

We note that not only the relation between the nodes is direct, but also the weights of the Gauss and Szegö formulas are the same (or related by a factor 2 for the end points).

The outline of the paper is as follows. In Section 2 we will derive the necessary properties of weights and nodes of a Szegő quadrature formula if the weight $\omega(\theta)$ is assumed to be an even function. Section 3 gives the relation between the formulas $I_{n}$ and $\tilde{I}_{2 n}$ for $\tau=1$, thus the relation between the Gauss and Szegö formulas. The relation between $\tilde{I}_{2 n}$ with $\tau=-1$ and the Gauss-Lobatto formulas is given in Section 4, and also the relation between $\tilde{I}_{2 n+1}$ with $\tau=1$ or $\tau=-1$ and the Gauss-Radau formulas is given here. Finally in Section 5 we give some illustrative examples of the relationships that were derived.

Before starting the paper, we introduce some notation. The unit circle in the complex plane $\mathbb{C}$ is denoted by $\mathbb{T}=\{z \in \mathbb{C}:|z|=1\}$. We denote the space of Laurent polynomials as $\Lambda_{m, n}=$ $\left\{\sum_{k=m}^{n} a_{k} z^{k}: a_{k} \in \mathbb{C}, m, \leq k \leq n\right\}$, and the space of polynomials of degree at most $n$ as $\Pi_{n}=\Lambda_{0, n}$. For any function $f$, the para-hermitian conjugate is denoted by a substar: $f_{*}(z)=\overline{f(1 / \bar{z})}$. If $\varphi_{n} \in$ $\Pi_{n} \backslash \Pi_{n-1}$, then the superstar conjugate is defined as $\varphi_{n}^{*}(z)=z^{n} \varphi_{n *}(z)=z^{n} \overline{\varphi_{n}(1 / \bar{z})}$. Note that the leading coefficient $\kappa_{n}$ of a polynomial $\varphi_{n}(z)=\kappa_{n} z^{n}+$ lower degree terms $\in \Pi_{n}$ can be recovered as $\kappa_{n}=\varphi_{n}^{*}(0)$. In this paper, we reserve the notation $\varphi_{n}$ to denote the $n$th monic orthonormal polynomial.

\section{Symmetric weight functions}

In this section we are concerned with Szegö quadrature formulas associated with a symmetric weight function, i.e., a function $\omega(\theta)$ defined on $[-\pi, \pi]$, such that $\omega(\theta)>0$ a.e., and

$$
\omega(-\theta)=\omega(\theta), \quad \theta \in[-\pi, \pi]
$$


Let $\left\{\varphi_{n}\right\}$ be the corresponding sequence of monic orthogonal Szegö polynomials. Here orthogonality should be understood with respect to the inner product induced by $\omega(\theta)$, i.e.,

$$
\langle f, g\rangle_{\omega}=\int_{-\pi}^{\pi} f\left(e^{i \theta}\right) \overline{g\left(e^{i \theta}\right)} \omega(\theta) \mathrm{d} \theta .
$$

In this case, it is known that all the coefficients of the powers of $z$ in $\varphi_{n}(z)$ are real for each $n=0,1, \ldots$. These Szegö polynomials satisfy the recurrence relation

$$
\varphi_{n+1}(z)=z \varphi_{n}(z)+\rho_{n} \varphi_{n}^{*}(z), \quad n \geq 0, \varphi_{0}=1,
$$

where the $\rho_{n}=\varphi_{n+1}(0)$ is a reflection coefficient or Schur parameter. Consequently, the reflection coefficients are real if the $\varphi_{k}$ have real coefficients. Replacing $\rho_{k}$ by $-\rho_{k}$ in the recurrence relation, we get another sequence of polynomials $\psi_{k}$, the so called associated polynomials of the second kind. Obviously the latter also have real coefficients if the $\rho_{k}$ are real. Finally, we recall that the zeros of the Szegö polynomials lie in the open unit disk $\mathbb{D}$, hence they will be real or appear in complex conjugate pairs if the coefficients are real.

Let us now consider the para-orthogonal polynomials

$$
B_{n}(z, \tau)=\varphi_{n}(z)+\tau \varphi_{n}^{*}(z), \quad|\tau|=1 .
$$

First take $\tau=1$. We know that (see e.g. [6]) all the zeros of $B_{n}(z, \tau),(|\tau|=1)$ are simple and lie on the unit circle $\mathbb{T}$. Since $\varphi_{n}(1)=\varphi_{n}^{*}(1) \neq 0$, the point $z=1$ cannot be a zero of $B_{n}(z, 1)$. Furthermore, the coefficients of $B_{n}(z, 1)$ are real. Thus, for $n$ even, all the zeros of $B_{n}(z, 1)$ appear on $\mathbb{T}$ in complex conjugate pairs. For $n$ odd, the only real zero is $z=-1$ and the $n-1$ remaining zeros are complex conjugate pairs and lie on $\mathbb{T}$.

Take now $\tau=-1$. Then $B_{n}(z,-1)=\varphi_{n}(z)-\varphi_{n}^{*}(z)$. Clearly, for each $n=1,2, \ldots$, the point $z=1$ is a zero. If $n$ is even, then $z=-1$ should also be a zero (observe that $\varphi_{n}(-1)=\varphi_{n}^{*}(-1)$ ). If $n$ is odd, then $z=1$ is the only real zero. In both cases, the remaining zeros appear again on $\mathbb{T}$ in conjugate pairs.

Let us next consider the integral

$$
\tilde{I}_{\omega}(f)=\int_{-\pi}^{\pi} f\left(e^{i \theta}\right) \omega(\theta) \mathrm{d} \theta
$$

to be approximated by an $n$-point Szegö quadrature formula

$$
\tilde{I}_{n}(f)=\sum_{j=1}^{n} A_{j} f\left(z_{j}\right)
$$

where as is known (see [1]) the nodes $\left\{z_{j}\right\}_{j=1}^{n}$ are the zeros of $B_{n}(z, \tau),(\tau \in \mathbb{T})$ and the weights $\left\{A_{j}\right\}_{j=1}^{n}$ are positive and can be expressed as

$$
A_{j}=-\frac{1}{2 z_{j}} \frac{A_{n}\left(z_{j}, \tau\right)}{B_{n}^{\prime}\left(z_{j}, \tau\right)}
$$

where $A_{n}(z, \tau)=\psi_{n}(z)-\tau \psi_{n}^{*}(z)$, with $\psi_{n}$ the $n$th associated polynomial of the second kind. If we take $\tau= \pm 1$, then both $A_{n}$ and $B_{n}$ have real coefficients.

Denote by $\tilde{A}_{j}$ the coefficient in formula $(2.3)$ corresponding to the node $\bar{z}_{j}$, where $z_{j}$ is a zero of $B_{n}(z, \pm 1)$. Then

$$
\tilde{A}_{j}=-\frac{1}{2 \bar{z}_{j}} \frac{A_{n}\left(\bar{z}_{j}, \pm 1\right)}{B_{n}^{\prime}\left(\bar{z}_{j}, \pm 1\right)}=-\frac{1}{2 \bar{z}_{j}} \frac{\overline{A_{n}\left(z_{j}, \pm 1\right)}}{\overline{B_{n}^{\prime}\left(z_{j}, \pm 1\right)}}=\bar{A}_{j}
$$


Since $A_{j}>0$, it follows that $\tilde{A}_{j}=\bar{A}_{j}=A_{j}$. Thus we have proved the following proposition.

Proposition 2.1. Let $\omega(\theta)$ be a symmetric weight function on $[-\pi, \pi]$ and let $\tilde{I}_{n}(f)=\sum_{j=1}^{n} A_{j} f\left(z_{j}\right)$ be the n-point Szegö quadrature formula, taking as nodes the zeros of the para-orthogonal polynomial $B_{n}(z, \tau)=\varphi_{n}(z)+\tau \varphi_{n}^{*}(z),|\tau|=1$. Then

1. If $\tau=+1$, then
(a) If $n$ is even: $\tilde{I}_{n}(f)=\sum_{j=1}^{n / 2} A_{j}\left[f\left(z_{j}\right)+f\left(\bar{z}_{j}\right)\right]$.
(b) If $n$ is odd: $\tilde{I}_{n}(f)=B f(-1)+\sum_{j=1}^{(n-1) / 2} A_{j}\left[f\left(z_{j}\right)+f\left(\bar{z}_{j}\right)\right]$.

2. If $\tau=-1$, then
(a) If $n$ is even: $\tilde{I}_{n}(f)=A f(1)+B f(-1)+\sum_{j=1}^{(n-2) / 2} A_{j}\left[f\left(z_{j}\right)+f\left(\bar{z}_{j}\right)\right]$.
(b) If $n$ is odd: $\tilde{I}_{n}(f)=A f(1)+\sum_{j=1}^{(n-1) / 2} A_{j}\left[f\left(z_{j}\right)+f\left(\bar{z}_{j}\right)\right]$.

All the coefficients $A, B$, and $A_{k}$ are positive.

Remark 2.2. Because of symmetry of the weight function $\omega(\theta)$, we have only to compute half of the nodes and coefficients in formula (2.3).

\section{Gaussian quadrature formulas}

Let $\sigma(x)$ be a weight function on the interval $[-1,1]$. We can define a symmetric weight function $\omega(\theta)$ on $[-\pi, \pi]$ by

$$
\omega(\theta)=\sigma(\cos \theta)|\sin \theta|, \quad \theta \in[-\pi, \pi] .
$$

Setting $z=e^{i \theta}, x=\frac{1}{2}\left(z+z^{-1}\right)=\cos \theta$, we can write

$$
\int_{-1}^{+1} F(x) \sigma(x) \mathrm{d} x=-\int_{-\pi}^{0} F(\cos \theta) \sigma(\cos \theta) \sin \theta \mathrm{d} \theta .
$$

On the other hand, setting $\xi=-\theta$, it follows that

$$
\int_{-1}^{+1} F(x) \sigma(x) \mathrm{d} x=\int_{0}^{\pi} F(\cos \xi) \sigma(\cos \xi) \sin \xi \mathrm{d} \xi .
$$

Thus

$$
2 \int_{-1}^{+1} F(x) \sigma(x) \mathrm{d} x=\left[\int_{-\pi}^{0}+\int_{0}^{\pi}\right] F(\cos \theta) \sigma(\cos \theta)|\sin \theta| \mathrm{d} \theta=\int_{-\pi}^{\pi} F(\cos \theta) \sigma(\cos \theta)|\sin \theta| \mathrm{d} \theta,
$$

or equivalently

$$
\int_{-1}^{+1} F(x) \sigma(x) \mathrm{d} x=\int_{-\pi}^{\pi} f\left(e^{i \theta}\right) \omega(\theta) \mathrm{d} \theta
$$

where

$$
f\left(e^{i \theta}\right)=\frac{1}{2} F\left(\frac{e^{i \theta}+e^{-i \theta}}{2}\right) .
$$

Let $\left\{p_{n}(x)\right\}$ be the sequence of monic orthogonal polynomials for the weight function $\sigma(x)$ on $[-1,1]$. A connection between orthogonal polynomials on the unit circle and orthogonal polynomials on a real interval was established by Szegő in his famous book [8, p. 294]. 
Theorem 3.1. Let $\sigma(x)$ be a weight function in the interval $[-1,1]$ and let $\omega(\theta)$ be defined by $\omega(\theta)=$ $\sigma(\cos \theta)|\sin \theta|, \theta \in[-\pi, \pi]$. Furthermore, let $\left\{p_{n}\right\}$ and $\left\{q_{n}\right\}$ be the sequences of monic orthogonal polynomials for $\sigma(x)$ and $\left(1-x^{2}\right) \sigma(x)$ on $[-1,1]$ respectively. Finally, let $\left\{\varphi_{n}(z)\right\}$ be the sequence of monic orthogonal Szegö polynomials associated with $\omega(\theta)$. Then, by writing $x=\frac{1}{2}\left(z+z^{-1}\right), z=e^{i \theta}$, we have for $n \geq 1$

$$
\begin{aligned}
& p_{n}(x)=\left\{2^{n}\left(1+\varphi_{2 n}(0)\right)\right\}^{-1}\left\{z^{-n} \varphi_{2 n}(z)+z^{n} \varphi_{2 n}\left(z^{-1}\right)\right\} \\
& q_{n}(x)=\left\{2^{n}\left(1-\varphi_{2 n+2}(0)\right)\right\}^{-1} \frac{\left\{z^{-n-1} \varphi_{2 n+2}(z)-z^{n+1} \varphi_{2 n+2}\left(z^{-1}\right)\right\}}{z-z^{-1}} .
\end{aligned}
$$

From (3.3) we see that the expression $z^{-n} \varphi_{2 n}(z)+z^{n} \varphi_{2 n}\left(z^{-1}\right)$ can be written as

$$
z^{-n} \varphi_{2 n}(z)+z^{n} \varphi_{2 n}\left(z^{-1}\right)=\frac{\varphi_{2 n}(z)+z^{2 n} \varphi_{2 n *}(z)}{z^{n}}=\frac{B_{2 n}(z, 1)}{z^{n}} .
$$

By Proposition 2.1 the zeros of $B_{2 n}(z, 1)$ appear in conjugate pairs. Let $z_{1}, \ldots, z_{n}, \bar{z}_{1}, \ldots, \bar{z}_{n}$ be these zeros. Setting $z_{j}=e^{i \theta_{j}}, j=1, \ldots, n$, the zeros of $p_{n}(x)$ are given by

$$
x_{j}=\cos \theta_{j}, \quad j=1, \ldots, n .
$$

Similarly, by (3.4), we can write

$$
\frac{z^{-n-1} \varphi_{2 n+2}(z)-z^{n+1} \varphi_{2 n+2}\left(z^{-1}\right)}{z-z^{-1}}=\frac{\varphi_{2 n+2}(z)-z^{2 n+2} \varphi_{2 n+2 *}(z)}{z^{n}\left(z^{2}-1\right)}=\frac{B_{2 n+2}(z,-1)}{z^{n}\left(z^{2}-1\right)} .
$$

By Proposition 2.1 we know that $z= \pm 1$ are zeros of $B_{2 n+2}(z,-1)$ and that the remaining zeros appear in conjugate pairs on $\mathbb{T}$. Let $z_{1}, \ldots, z_{n}, \bar{z}_{1}, \ldots, \bar{z}_{n}$ be these zeros. Letting $z_{j}=e^{i \theta_{j}}, j=1, \ldots, n$, then the zeros of $q_{n}(x)$ are given by

$$
x_{j}=\cos \theta_{j}, \quad j=1, \ldots, n .
$$

On the other hand, one knows that the integral $\int_{-1}^{1} F(x) \sigma(x) \mathrm{d} x$ can be approximated by an $n$-point Gauss-Christoffel quadrature formula. More precisely,

$$
I_{\sigma}(F)=\int_{-1}^{+1} F(x) \sigma(x) \mathrm{d} x=\sum_{j=1}^{n} \lambda_{j} F\left(x_{j}\right)+E_{n}(F),
$$

such that $E_{n}(F)=0$ for all $F \in \Pi_{2 n-1}$. The nodes $\left\{x_{j}\right\}_{j=1}^{n}$ are the zeros of $p_{n}(x)$ (hence given by (3.5)) and the weights $\lambda_{j}$ (Christoffel numbers) are positive.

A connection between Gauss and Szegő quadrature formulas is given in the following theorem.

Theorem 3.2. Let $I_{n}(F)=\sum_{j=1}^{n} \lambda_{j} F\left(x_{j}\right)$ be the n-point Gauss formula for $I_{\sigma}(F)=\int_{-1}^{1} F(x) \sigma(x) \mathrm{d} x$. Set $x_{j}=\cos \theta_{j}$ and define $\left\{z_{j}\right\}_{j=1}^{2 n}$ and $\left\{A_{j}\right\}_{j=1}^{2 n}$ by means of

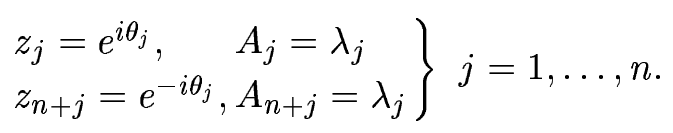

Then $\tilde{I}_{2 n}(f)=\sum_{j=1}^{2 n} A_{j} f\left(z_{j}\right)=\sum_{j=1}^{n} A_{j}\left[f\left(z_{j}\right)+f\left(\bar{z}_{j}\right)\right]$ coincides with the $2 n$-point Szegö quadrature formula for $\tilde{I}_{\omega}(f)=\int_{-\pi}^{\pi} f\left(e^{i \theta}\right) \mathrm{d} \omega(\theta)$, where $\omega(\theta)=\sigma(\cos \theta)|\sin \theta|$ when taking as nodes the zeros of $B_{2 n}(z, 1)$. 
Proof. From the characterization theorem (see e.g. [1]) for Szegö quadrature formulas, we must prove that $\tilde{I}_{2 n}(L)=\tilde{I}_{\omega}(L)=\int_{-\pi}^{\pi} L\left(e^{i \theta}\right) \omega(\theta) \mathrm{d} \theta$, for any $L \in \Lambda_{-(2 n-1),(2 n-1)}$, or equivalently,

$$
\tilde{I}_{2 n}\left(z^{k}\right)=\tilde{I}_{\omega}\left(z^{k}\right), \quad-(2 n-1) \leq k \leq 2 n-1 .
$$

We shall restrict ourselves to $k \geq 0$ (the same can be done for $k<0$ ). By (3.7) we can write

$$
\tilde{I}_{2 n}\left(z^{k}\right)=\sum_{j=1}^{n} A_{j}\left[z_{j}^{k}+\bar{z}_{j}^{k}\right]=2 \sum_{j=1}^{n} A_{j} \cos k \theta_{j} .
$$

We can write $\cos k \theta=T_{k}(\cos \theta), T_{k}(x)$ being the $k$ th Chebyshev polynomial of the first kind. Since $0 \leq|k| \leq 2 n-1$, we have

$$
\tilde{I}_{2 n}\left(z^{k}\right)=2 \sum_{j=1}^{n} A_{j} T_{k}\left(\cos \theta_{j}\right)=2 \sum_{j=1}^{n} A_{j} T_{k}\left(x_{j}\right)=2 \int_{-1}^{+1} T_{k}(x) \sigma(x) \mathrm{d} x .
$$

By (3.2), the last integral can be written as $2 \int_{-\pi}^{\pi} p\left(e^{i \theta}\right) \omega(\theta) \mathrm{d} \theta$, where $p\left(e^{i \theta}\right)=\frac{1}{2} T_{k}\left(\frac{e^{i \theta}+e^{-i \theta}}{2}\right)=$ $\frac{1}{2} T_{k}(\cos \theta)=\frac{1}{2} \cos k \theta$.

In short, we arrive at $\tilde{I}_{2 n}\left(z^{k}\right)=\int_{-\pi}^{\pi} \cos k \theta \omega(\theta) \mathrm{d} \theta$.

On the other hand,

$$
\tilde{I}_{\omega}\left(z^{k}\right)=\int_{-\pi}^{\pi} e^{i k \theta} \omega(\theta) \mathrm{d} \theta=\int_{-\pi}^{\pi} \cos k \theta \omega(\theta) \mathrm{d} \theta+i \int_{-\pi}^{\pi} \sin k \theta \omega(\theta) \mathrm{d} \theta .
$$

Since $\omega(\theta)$ is symmetric, the second integral is zero. Thus we conclude that $\tilde{I}_{2 n}\left(z^{k}\right)=\int_{-\pi}^{\pi} e^{i k \theta} \omega(\theta) \mathrm{d} \theta$, $0 \leq k \leq 2 n-1$. Using a similar argument for negative powers of $z$, the proof follows.

A converse result can also be given. Indeed, one has

Theorem 3.3. Let $\tilde{I}_{2 n}(f)=\sum_{j=1}^{2 n} A_{j} f\left(z_{j}\right)$ be the $2 n$-point Szegö formula for $\tilde{I}_{\omega}(f)$ with $\omega(\theta)=$ $\sigma(\cos \theta)|\sin \theta|$, and whose nodes are the zeros of $B_{2 n}(z, 1)=\varphi_{2 n}(z)+\varphi_{2 n}^{*}(z)$. Set $z_{n+j}=\bar{z}_{j}$ and $z_{j}=$ $e^{i \theta_{j}}, j=1, \ldots, n$. Then, when taking $x_{j}=\cos \theta_{j}, j=1, \ldots, n$, the formula $I_{n}(F)=\sum_{j=1}^{n} A_{j} F\left(x_{j}\right)$ coincides with the n-point Gauss formula for $I_{\sigma}(F)=\int_{-1}^{1} F(x) \sigma(x) \mathrm{d} x$.

Proof. We have to prove that $I_{n}(P)=I_{\sigma}(P)$ for any polynomial $P \in \Pi_{2 n-1}$ or equivalently

$$
I_{n}\left(x^{k}\right)=I_{\sigma}\left(x^{k}\right), \quad k=0,1, \ldots, 2 n-1 .
$$

Notice that with $x=\left(z+z^{-1}\right) / 2$

$$
x^{k}=\left(\frac{z+z^{-1}}{2}\right)^{k}=p(z)+p\left(z^{-1}\right)=L(z) \in \Lambda_{-k, k},
$$

where $p$ is a polynomial of degree $k$ with real coefficients. Therefore

$$
\overline{L(z)}=L(\bar{z})=L\left(z^{-1}\right)=L(z), \quad z=e^{i \theta} .
$$

Since we know that $A_{j}=A_{n+j}$ and $z_{n+j}=\bar{z}_{j}, j=1, \ldots, n$, we get

$$
\begin{aligned}
I_{n}\left(x^{k}\right) & =\sum_{j=1}^{n} A_{j} x_{j}^{k}=\sum_{j=1}^{n} A_{j} L\left(z_{j}\right)=\sum_{j=1}^{n}\left(\frac{A_{j}+A_{j}}{2}\right) L\left(z_{j}\right) \\
& =\frac{1}{2} \sum_{j=1}^{2 n} A_{j} L\left(z_{j}\right)=\frac{1}{2} \int_{-\pi}^{\pi} L\left(e^{i \theta}\right) \omega(\theta) \mathrm{d} \theta
\end{aligned}
$$


since $L \in \Lambda_{-k, k} \subset \Lambda_{-(2 n-1), 2 n-1}$.

On the other hand, by (3.2),

$$
\int_{-1}^{+1} x^{k} \sigma(x) \mathrm{d} x=\frac{1}{2} \int_{-\pi}^{\pi}(\cos \theta)^{k} \sigma(\cos \theta)|\sin \theta| \mathrm{d} \theta=\frac{1}{2} \int_{-\pi}^{\pi} L\left(e^{i \theta}\right) \omega(\theta) \mathrm{d} \theta=I_{n}\left(x^{k}\right),
$$

for $k=0,1, \ldots, 2 n-1$, and this concludes the proof.

Remark 3.4. Set $p_{n}(x)=\prod_{j=1}^{n}\left(x-x_{j}\right)$, i.e., the nodal polynomial which one knows to coincide with the $n$th monic orthogonal polynomial for the weight $\sigma(x)$. Since $x_{j}=\cos \theta_{j}$, and $z_{j}=e^{i \theta_{j}}, j=1, \ldots, n$, one can write

$$
\begin{aligned}
B_{2 n}(z, 1) & =\varphi_{2 n}(z)+\varphi_{2 n}^{*}(z) \\
& =\left(1+\varphi_{2 n}(0)\right) \prod_{j=1}^{n}\left(z-z_{j}\right)\left(z-\bar{z}_{j}\right) \\
& =\left(1+\varphi_{2 n}(0)\right) z^{n} \prod_{j=1}^{n}\left(z-z_{j}\right)\left(1-\bar{z}_{j} / z\right) .
\end{aligned}
$$

But if $x=\left(z+z^{-1}\right) / 2$, then $\left(z-z_{j}\right)\left(1-\bar{z}_{j} / z\right)=\left(z+z^{-1}\right)-\left(z_{j}+\bar{z}_{j}\right)=2\left(x-x_{j}\right)$. Therefore $\varphi_{2 n}(z)+\varphi_{2 n}^{*}(z)=\left(1+\varphi_{2 n}(0)\right) z^{n} 2^{n} p_{n}(x)$, with

$$
\begin{aligned}
p_{n}(x) & =\frac{z^{-n}}{\left(1+\varphi_{2 n}(0)\right) 2^{n}}\left[\varphi_{2 n}(z)+z^{2 n} \varphi_{2 n}\left(z^{-1}\right)\right] \\
& =\frac{1}{\left(1+\varphi_{2 n}(0)\right) 2^{n}}\left[z^{-n} \varphi_{2 n}(z)+z^{n} \varphi_{2 n}\left(z^{-1}\right)\right] .
\end{aligned}
$$

Thus we see that formula (3.3) has been deduced in a different and rather simple way, just making use of the connection between quadratures.

\section{Gauss-Lobatto and Gauss-Radau quadrature}

We have seen that the $n$-point Gauss quadrature formula could be obtained from the $2 n$-point Szegö formula taking as nodes the zeros of the $(2 n)$ th para-orthogonal polynomial

$$
B_{2 n}(z, 1)=\varphi_{2 n}(z)+\varphi_{2 n}^{*}(z) .
$$

Now, we might wonder what role could be played by other Szegö quadrature formulas coming from other para-orthogonal functions closely related to (4.1) such as $\varphi_{2 n}(z)-\varphi_{2 n}^{*}(z)$ for example, or $\varphi_{2 n+1}(z) \pm \varphi_{2 n+1}^{*}(z)$. A connection with the well known Gauss-Lobatto and Gauss-Radau formulas will be established in this way.

It is known (see e.g., [7, p. 170]) that if quadrature formulas for $I_{\sigma}(F)=\int_{-1}^{+1} F(x) \sigma(x) \mathrm{d} x$ with preassigned nodes $a_{1}, \ldots, a_{m}$ are required, i.e., formulas of the type

$$
I_{\sigma}(F)=\sum_{j=1}^{m} B_{j} F\left(a_{j}\right)+\sum_{j=1}^{n} \lambda_{j} F\left(x_{j}\right)+\tilde{E}_{n}(F) \equiv J_{n}(F)+\tilde{E}_{n}(F),
$$

then the highest degree of precision which can be achieved by $(4.2)$ is $2 n+m-1$, i.e., $\tilde{E}_{n}(F)=0$ for any $F \in \Pi_{2 n+m-1}$.

This is described in the following theorem.

Theorem 4.1. The quadrature formula $J_{n}(F)$ given in (4.2) is exact in $\Pi_{2 n+m-1}$ if and only if 
1. $J_{n}(F)$ is interpolatory in $\Pi_{n+m-1}$.

2. The nodes $\left\{x_{j}\right\}_{j=1}^{n}$ are the zeros of the $n$th orthogonal polynomial for the weight function $\nu(x) \sigma(x)$ with $\nu(x)=\left(x-a_{1}\right) \cdots\left(x-a_{m}\right)$.

Furthermore, if $\nu\left(x_{k}\right) \neq 0$ for $k=1, \ldots, n$, then both sets of weights $\left\{B_{j}\right\}_{j=1}^{m}$ and $\left\{\lambda_{j}\right\}_{j=1}^{n}$ are positive.

Notice that the position of the nodes $a_{i}$ is important if we want to guarantee the new weight function $\nu(x) \sigma(x)$ to be positive.

Assume now $m=2, a_{1}=-1$ and $a_{2}=1$ (Gauss-Lobatto formula). The nodes $\left\{x_{k}\right\}$ are the zeros of the $n$th orthogonal polynomial for the weight $\nu(x) \sigma(x)=\left(1-x^{2}\right) \sigma(x)$. From Theorem 3.1, we see that the nodes are given in terms of the zeros of the para-orthogonal polynomial $B_{2 n+2}(z,-1)=$ $\varphi_{2 n+2}(z)-\varphi_{2 n+2}^{*}(z)$ which by Proposition 2.1, part 2, has got $2 n$ zeros in complex conjugate pairs on $\mathbb{T}$ together with $z= \pm 1$. Let $z_{1}, \ldots, z_{2 n}$ be the $2 n$ complex zeros. By writing $z_{n+j}=\bar{z}_{j}=e^{-i \theta_{j}}$, $j=1, \ldots, n$, we see from (3.4) that the zeros of $q_{n}(x)$ and hence the nodes $\left\{x_{k}\right\}$ in formula (4.2) are given by $x_{j}=\cos \theta_{j}, j=1, \ldots, n$.

Proceeding as in Theorem 3.2, we can prove the following

Theorem 4.2. Let $A F(1)+B F(-1)+\sum_{j=1}^{n} \lambda_{j} F\left(x_{j}\right)$ be the n-point Gauss-Lobatto formula for $I_{\sigma}(F)$, $\sigma(x)$ being a weight function on $[-1,1]$. Let $x_{j}=\cos \theta_{j}, z_{j}=e^{i \theta_{j}}$ and $A_{j}=\lambda_{j}, j=1, \ldots, n$. Then $2 A f(1)+2 B f(-1)+\sum_{j=1}^{n} A_{j}\left[f\left(z_{j}\right)+f\left(\bar{z}_{j}\right)\right]$ represents the $(2 n+2)$-point Szegö formula for $\tilde{I}_{\omega}(f)$ with $\omega(\theta)=\sigma(\cos \theta)|\sin \theta|, \theta \in[-\pi, \pi]$, whose nodes are the zeros of $B_{2 n+2}(z,-1)$.

Similarly it follows that

Theorem 4.3. Let $\tilde{I}_{2 n+2}(f)=\sum_{j=1}^{2 n+2} A_{j} f\left(z_{j}\right)$ be the $(2 n+2)$-point Szegö formula for $\tilde{I}_{\omega}(f)$ taking as nodes the zeros of $B_{2 n+2}(z,-1)=\varphi_{2 n+2}(z)-\varphi_{2 n+2}^{*}(z)$. Set $z_{2 n+1}=1$ and $z_{2 n+2}=-1$ and $z_{n+j}=\bar{z}_{j}$, $j=1, \ldots, n$ with $z_{j}=e^{i \theta_{j}}, \theta_{j} \notin\{-\pi, 0\}$. Take $A=A_{2 n+1}$ and $B=A_{2 n+2}$. Then

$$
J_{n}(F)=\frac{A}{2} F(1)+\frac{B}{2} F(-1)+\sum_{j=1}^{n} A_{j} F\left(x_{j}\right), \quad x_{j}=\cos \theta_{j}, \quad j=1, \ldots, n,
$$

coincides with the n-point Gauss-Lobatto formula for $I_{\sigma}(F)$.

Proof. We have to prove that $J_{n}\left(x^{k}\right)=I_{\sigma}\left(x^{k}\right), k=0,1, \ldots, 2 n+1$. Proceeding as in the proof of Theorem 3.3, one can write

$$
\begin{aligned}
J_{n}\left(x^{k}\right) & =\frac{A}{2}+\frac{B(-1)^{k}}{2}+\sum_{j=1}^{n} A_{j} x_{j}^{k} \\
& =\frac{A}{2}+\frac{B(-1)^{k}}{2}+\sum_{j=1}^{n} A_{j}\left[p\left(z_{j}\right)+p\left(\bar{z}_{j}\right)\right]
\end{aligned}
$$

where $p(z)$ is a polynomial of degree $k$ with real coefficients. More precisely,

$$
\begin{aligned}
& p(z)=\frac{1}{2^{k}} \sum_{j=0}^{(k-1) / 2}\left(\begin{array}{l}
k \\
j
\end{array}\right) z^{k-2 j} \quad \text { if } k \text { is odd } \\
& p(z)=\frac{1}{2^{k}} \sum_{j=0}^{k / 2-1}\left(\begin{array}{l}
k \\
j
\end{array}\right) z^{k-2 j}+\frac{1}{2^{k}}\left(\begin{array}{c}
k \\
k / 2
\end{array}\right) \quad \text { if } k \text { is even. }
\end{aligned}
$$


Setting $L(z)=p(z)+p(\bar{z}) \in \Lambda_{-k, k} \subset \Lambda_{-(2 n+1), 2 n+1}$ we have $L(\bar{z})=L\left(z^{-1}\right)=L(z)$. Thus we can write

$$
J_{n}\left(x^{k}\right)=\frac{A}{2}+\frac{B(-1)^{k}}{2}+\frac{1}{2} \sum_{j=1}^{2 n} A_{j} L\left(z_{j}\right) .
$$

To fix the ideas, assume first that $k$ is odd. Then

$$
L(1)=2 p(1)=\frac{2}{2^{k}} \sum_{j=0}^{(k-1) / 2}\left(\begin{array}{l}
k \\
j
\end{array}\right)=\frac{(1+1)^{k}}{2^{k}}=1 .
$$

Similarly $L(-1)=-1$. Thus

$$
J_{n}\left(x^{k}\right)=\frac{1}{2}\left[A L(1)+B L(-1)+\sum_{j=1}^{2 n} A_{j} L\left(z_{j}\right)\right]=\frac{1}{2} \tilde{I}_{2 n+2}(L)=\frac{1}{2} \int_{-\pi}^{\pi} L\left(e^{i \theta}\right) \omega(\theta) \mathrm{d} \theta
$$

since $L \in \Lambda_{-(2 n+1), 2 n+1}$. Now by (3.2), the proof easily follows for the case $k$ odd. For $k$ even, the proof is completely analogous, except that $L(-1)=1$. Thus the proof is complete.

Finally we have the following theorem.

Theorem 4.4. Let $\sigma(x)$ be a weight function on $[-1,1]$. Consider the weight functions associated with $\sigma(x)$ :

$$
\sigma^{+}(x)=(1+x) \sigma(x) \quad \text { and } \quad \sigma^{-}(x)=(1-x) \sigma(x), \quad x \in[-1,1] .
$$

Let $\left\{Q_{n}^{+}(x)\right\}$ and $\left\{Q_{n}^{-}(x)\right\}$ be the sequences of monic orthogonal polynomials for $\sigma^{+}(x)$ and $\sigma^{-}(x)$ respectively. Define the weight function $\omega(\theta)$ on $[-\pi, \pi]$ by $\omega(\theta)=\sigma(\cos \theta)|\sin \theta|$, and let $\left\{\varphi_{n}(z)\right\}$ be the sequence of monic Szegö polynomials for $\omega(\theta)$. Then, setting $x=\left(z+z^{-1}\right) / 2$, it holds that

$$
Q_{n}^{ \pm}(x)=\frac{1}{2^{n}\left(1 \pm \varphi_{2 n+1}(0)\right)}\left[\frac{z^{-n} \varphi_{2 n+1}(z) \pm z^{n+1} \varphi_{2 n+1}\left(z^{-1}\right)}{z \pm 1}\right]
$$

Proof. Let us consider the $(2 n+1)$-point Szegő formula $\tilde{I}_{2 n+1}(f)$ for $\tilde{I}_{\omega}(f)=\int_{-\pi}^{\pi} f\left(e^{i \theta}\right) \omega(\theta) \mathrm{d} \theta$, with nodes corresponding to the zeros of the para-orthogonal polynomial $B_{2 n+1}(z, 1)=\varphi_{2 n+1}(z)+\varphi_{2 n+1}^{*}(z)$. We know that $z=-1$ is a zero of $B_{2 n+1}(z, 1)$. We use the ordering such that $z_{j}=e^{i \theta_{j}}$ and $z_{n+j}=e^{-i \theta_{j}}$ are the remaining $2 n$ complex conjugate zeros. Then we have

$$
\tilde{I}_{2 n+1}(f)=A f(-1)+\sum_{j=1}^{n} A_{j}\left[f\left(z_{j}\right)+f\left(\bar{z}_{j}\right)\right] .
$$

Proceeding as in the proof of Theorem 4.3, one can derive that $\left(x_{j}=\cos \theta_{j}\right)$

$$
J_{n}(F)=\frac{A}{2} F(-1)+\sum_{j=1}^{n} A_{j} F\left(x_{j}\right)=I_{\sigma}(F)=\int_{-1}^{+1} F(x) \sigma(x) \mathrm{d} x,
$$

for all $F \in \Pi_{2 n}$. Thus, setting $Q_{n}^{+}(x)=\prod_{j=1}^{n}\left(x-x_{j}\right)$, we know by Theorem 4.2 that $Q_{n}^{+}(x)$ coincides with the $n$th monic orthogonal polynomial for the weight function $(1+x) \sigma(x)=\sigma^{+}(x)$. On the other hand, using the formulas of Remark 3.4,

$$
B_{2 n+1}(z, 1)=\left(1+\varphi_{2 n+1}(0)\right)(z+1) \prod_{j=1}^{n}\left(z-z_{j}\right)\left(z-\bar{z}_{j}\right)
$$




$$
\begin{aligned}
& =\left(1+\varphi_{2 n+1}(0)\right) z^{n}(z+1) \prod_{j=1}^{n}\left(z-z_{j}\right)\left(1-\bar{z}_{j} / z\right) \\
& =\left(1+\varphi_{2 n+1}(0)\right) z^{n} 2^{n}(z+1) Q_{n}^{+}(x),
\end{aligned}
$$

and (4.3) easily follows. The case $\sigma^{-}(x)$ is treated similarly.

We can now formulate the analog of Theorem 3.2 (Gauss) and Theorem 4.2 (Gauss-Lobatto) an analog theorem in the Gauss-Radau case.

Theorem 4.5. Let $\frac{A}{2} F( \pm 1)+\sum_{j=1}^{n} \lambda_{j} F\left(x_{j}\right)$ be the n-point Gauss-Radau formula for $I_{\sigma}(F), \sigma(x)$ being a weight function on $[-1,1]$. Let $x_{j}=\cos \theta_{j}, z_{j}=e^{i \theta_{j}}$ and $A_{j}=\lambda_{j}, j=1, \ldots, n$. Then $A f( \pm 1)+\sum_{j=1}^{n} A_{j}\left[f\left(z_{j}\right)+f\left(\bar{z}_{j}\right)\right]$ represents the $(2 n+1)$-point Szegö formula for $\tilde{I}_{\omega}(f)$ with $\omega(\theta)=$ $\sigma(\cos \theta)|\sin \theta|, \theta \in[-\pi, \pi]$, whose nodes are the zeros of $B_{2 n+1}(z, \pm 1)$.

The proof is as in the case of the Gauss or the Gauss-Lobatto formulas. We leave the details to the reader.

Remark 4.6. As shown in Sections 3 and 4, the connection between the Szegö quadrature formula on $\mathbb{T}$ and Gauss, Gauss-Lobatto, or Gauss-Radau quadrature formulas on the interval $[-1,1]$ has allowed us to deduce directly and in a rather simple way the well known relations between orthogonal polynomials on $\mathbb{T}$ and orthogonal polynomials on $[-1,1]$. Furthermore, as far as we know, relations as given in (4.3) are new and not given explicitly before. It should be mentioned though that they can also be obtained in other ways. For example $\sigma^{ \pm}$is the product of a positive measure and a polynomial of degree 1 which vanises in one of the end points. For such polynomial modifications of a measure, the given relation between the corresponding orthogonal polynomials can also be derived from the Christoffel formula for orthogonal polynomials [8, Sec. 2.5].

\section{Some examples}

In this section we apply the previous formulas, which were derived for a general positive weight $\sigma(x)$, in special cases more precisely the so-called Chebyshev weight functions, i.e., weight functions of the form $\sigma(x)=(1-x)^{\alpha}(1+x)^{\beta}$, where $\alpha, \beta \in\{ \pm 1 / 2\}$. So we consider 4 possible cases which are listed in Table 5.1 below.

Table 5.1

\begin{tabular}{|c|c|c|c|c|}
\hline case & $\alpha$ & $\beta$ & $\sigma(x)$ & $\omega(\theta)$ \\
\hline 1 & $\mid-1 / 2$ & $-1 / 2$ & $\frac{1}{\sqrt{1-x^{2}}}$ & 1 \\
\hline 2 & $1 / 2$ & $1 / 2$ & $\sqrt{1-x^{2}}$ & $\sin ^{2} \theta$ \\
\hline 3 & $1 / 2$ & $-1 / 2$ & $\sqrt{\frac{1-x}{1+x}}$ & $2 \sin ^{2} \frac{\theta}{2}$ \\
\hline 4 & $-1 / 2$ & $1 / 2$ & $\sqrt{\frac{1+x}{1-x}}$ & $2 \cos ^{2} \frac{\theta}{2}$ \\
\hline
\end{tabular}

The Chebyshev weights

The first case is obviously the simplest one because the weight $\omega(\theta)=1$. The orthogonal Szegö polynomials are just $\varphi_{n}(z)=z^{n}$ and the monic orthogonal polynomials $p_{n}(x)$ for case 1 are the 
Chebyshev polynomials $2^{-n} T_{n}(x)$. Using Theorem 3.1, we can construct the polynomials $q_{n}(x)$ for case 1 and these correspond to the polynomials $p_{n}(x)$ for case 2, which are of course the Chebyshev polynomials of the second kind $2^{-n} U_{n}(x)$. Next, using Theorem 4.4 for the weight of case 1 , we obtain the orthogonal polynomials $Q_{n}^{ \pm}(x)$ which coincide with the polynomials $p_{n}(x)$ for cases 3 and 4 . This results in the first column of Table 5.2 below. The computation of the $\varphi_{n}$ can be found in [2]. They

Table 5.2

The orthogonal and para-orthogonal polynomials

\begin{tabular}{|c|c|c|c|}
\hline case & $p_{n}(\cos \theta)$ & $\varphi_{n}(z)$ & $B_{n}(z, \tau)$ \\
\hline 1 & $\frac{1}{2^{n}} \cos n \theta$ & $\varphi_{n}(z)=z^{n}$ & $B_{n}(z, \pm 1)=z^{n} \pm 1$ \\
\hline 2 & $\frac{1}{2^{n}} \frac{\sin (n+1) \theta}{\sin \theta}$ & $\begin{array}{c}\varphi_{2 n}(z)=\frac{1}{n+1} \frac{1-(n+2) z^{2 n+2}+(n+1) z^{2 n+4}}{\left(1-z^{2}\right)^{2}} \\
\varphi_{2 n+1}(z)=z \varphi_{2 n}(z)\end{array}$ & $\begin{array}{c}B_{2 n}(z, 1)=\frac{n+2}{n+1} \frac{1-z^{2 n+2}}{1-z^{2}} \\
B_{2 n}(z,-1)=\frac{-n\left(1-z^{2 n+4}\right)+(n+2) z^{2}\left(1-z^{2 n}\right)}{(n+1)\left(1-z^{2}\right)^{2}} \\
B_{2 n+1}(z, \pm 1)=\frac{ \pm(n+1)\left(1-z^{2 n+4}\right)+(n+2) z\left(1-z^{2 n+2}\right)}{(n+1)\left(1-z^{2}\right)(1 \pm z)}\end{array}$ \\
\hline 3 & $\frac{1}{2^{n}} \frac{\sin \left(n+\frac{1}{2}\right) \theta}{\sin \frac{1}{2} \theta}$ & $\varphi_{n}(z)=\frac{1}{n+1} \frac{1-(n+2) z^{n+1}+(n+1) z^{n+2}}{(1-z)^{2}}$ & $\begin{array}{c}B_{n}(z, 1)=\frac{n+2}{n+1} \frac{1-z^{n+1}}{1-z} \\
B_{n}(z,-1)=\frac{1}{n+1} \frac{n\left(z^{n+2}-1\right)-(n+2) z\left(z^{n}-1\right)}{(1-z)^{2}}\end{array}$ \\
\hline 4 & $\frac{1}{2^{n}} \frac{\cos \left(n+\frac{1}{2}\right) \theta}{\cos \frac{1}{2} \theta}$ & $\varphi_{n}(z)=\frac{1}{n+1} \frac{(-1)^{n}+(n+2) z^{n+1}+(n+1) z^{n+2}}{(1+z)^{2}}$ & $\begin{array}{c}B_{n}\left(z,(-1)^{n}\right)=\frac{(-1)^{n}(n+2)}{n+1} \frac{1+(-1)^{n} z^{n+1}}{1+z} \\
B_{n}\left(z,(-1)^{n+1}\right)=\frac{1}{n+1} \frac{n\left(z^{n+2}-(-1)^{n}\right)+(n+2) z\left(z^{n}-(-1)^{n}\right)}{(1+z)^{2}}\end{array}$ \\
\hline
\end{tabular}

could also be derived by inverting the formulas (3.3) and (3.4) to compute $\varphi_{n}(z)$ from $p_{n}(x)$ and $q_{n}(x)$. See also [8, p. 295]. For our formulas, we only need explicitly the values at the origin: $\varphi_{n}(0)$. Of course the full expression is needed to find the combination giving the para-orthogonal polynomials $B_{n}(z, \tau)$. But also that has been done in [2] and this combination can be reduced to a closed form expression as shown in Table 5.2. Note that although these weight functions are really simple, closed form expressions for the Szegö polynomials are not found in the classical literature.

Once we know the para-orthogonal polynomials, we can find their zeros to give the nodes of the Szegö quadrature formulas, and hence also of the Gauss (G), Gauss-Lobatto $(G L)$ or Gauss-Radau formulas including the point $1(G R(1))$ or $-1(G R(-1))$. Of course, the nodes of the Gauss formula could also be found from the expressions for the polynomials $p_{n}(x)$ that we gave in Table 5.2. These nodes, along with the corresponding weights are listed in Table 5.3 for the cases where an explicit expression exists.

In the squares numbered [a-g], there is no explicit expression for the nodes, but some simple relations defining them can be obtained. Let us take for example case [a], the Gauss-Lobatto formula for the weight $\sigma(x)=\sqrt{1-x^{2}}$. Then we need the zeros of the para-orthogonal polynomial $B_{2 n+2}(z,-1)$ for $\omega(\theta)=\sin ^{2} \theta$. Using the substitution $z=e^{i \theta}$, then we can easily see that its numerator is proportional to

$$
(n+1) \sin (n+3) \theta-(n+3) \sin (n+1) \theta,
$$

which reduces after some trigonometric manipulation to the simple equation

$$
(n+2) \cos (n+2) \theta \sin \theta=\sin (n+2) \theta \cos \theta .
$$

The solutions of this equation include 0 and $\pi$ and in case $n$ is odd, one has to add $\pi / 2$. The other solutions are symmetric with respect to $\pi / 2$. They have to be computed numerically though. For $n$ odd they are close to positions $\pi / 2 \pm j \pi /(n+2), j=0, \ldots,(n-1) / 2$ and for $n$ even, $\pi / 2$ is not 
Table 5.3

The nodes and weights of the quadrature formulas

\begin{tabular}{|c|c|c|c|c|}
\hline cas & $\begin{array}{c}G \\
j=1, \ldots, n\end{array}$ & $\begin{aligned} & G L \\
= & 0, \ldots, n\end{aligned}$ & $\begin{array}{c}G R(1) \\
j=0, \ldots, n\end{array}$ & $\begin{aligned} & G R(-1) \\
= & 1, \ldots, n+1\end{aligned}$ \\
\hline 1 & $\left|\begin{array}{c}\theta_{j}=\frac{(2 j-1) \pi}{2 n} \\
\lambda_{j}=\frac{\pi}{n}\end{array}\right|$ & $\begin{aligned} \theta_{j} & =\frac{j \pi}{n+1} \\
\lambda_{j} & =\frac{\pi}{n+1}\end{aligned}$ & $\begin{aligned} \theta_{j} & =\frac{2 j \pi}{2 n+1} \\
\lambda_{j} & =\frac{2 \pi}{2 n+1}\end{aligned}$ & $\begin{array}{c}\theta_{j}=\frac{(2 j-1) \pi}{2 n+1} \\
\lambda_{j}=\frac{2 \pi}{2 n+1}\end{array}$ \\
\hline 2 & $\left|\begin{array}{c}\theta_{j}=\frac{j \pi}{n+1} \\
\lambda_{j}=\frac{\pi \omega\left(\theta_{j}\right)}{n+1}\end{array}\right|$ & {$[a]$} & {$[b]$} & {$[c]$} \\
\hline 3 & $\left|\begin{array}{c}\theta_{j}=\frac{2 j \pi}{2 n+1} \\
\lambda_{j}=\frac{2 \pi \omega\left(\theta_{j}\right)}{2 n+1}\end{array}\right|$ & {$[d]$} & {$[e]$} & $\begin{array}{c}\theta_{j}=\frac{j \pi}{n+1} \\
\lambda_{j}=\frac{\pi \omega\left(\theta_{j}\right)}{n+1}\end{array}$ \\
\hline 4 & $\left|\begin{array}{l}\theta_{j}=\frac{(2 j-1) \pi}{2 n+1} \\
\lambda_{j}=\frac{2 \pi \omega\left(\theta_{j}\right)}{2 n+1}\end{array}\right|$ & {$[f]$} & $\begin{array}{c}\theta_{j}=\frac{j \pi}{n+1} \\
\lambda_{j}=\frac{\pi \omega\left(\theta_{j}\right)}{n+1}\end{array}$ & {$[g]$} \\
\hline
\end{tabular}

a solution and the others are again symmetric and close to the points $\pi / 2 \pm(2 j+1) \pi /(2 n+4)$, $j=0, \ldots,(n-2) / 2$. Similarly, one obtains simple equations of this type to find the nodes of the quadrature formulas for the other cases $[\mathrm{b}-\mathrm{g}]$.

There is a lot of symmetry in the solutions for the other cases. For example, the nodes for case [e] correspond to the solutions of

$$
(2 n+1) \sin \left(\frac{2 n+3}{2} \theta\right)=(2 n+3) \sin \left(\frac{2 n+1}{2} \theta\right) .
$$

They are located in $\theta_{0}=0$ of course and the other points are very close to $\pi / 2+(2 j+1) \pi /(2 n+2)$, $j=-(n-1) / 2, \ldots, 0, \ldots,(n-1) / 2$ for $n$ odd and near $\pi / 2+j \pi /(n+1), j=-(n-2) / 2, \ldots, 0, \ldots, n / 2$ for $n$ even. The nodes for case [g] are the supplements of case [e], i.e., they are equal to $\pi$ minus the $\theta_{j}$ of case [e]. The other cases have nodes that can be derived from the the solutions of the equation

$$
(2 n+3) \tan \left(\frac{2 n+3}{2} \theta\right)=\tan \frac{\theta}{2}
$$

or equivalently from the equation

$$
(n+1) \sin (n+2) \theta+(n+2) \sin (n+1) \theta=0 .
$$

The solutions include $\theta_{0}=0$ and $\theta_{n+1}=\pi$. The other solutions are close to the positions $2 j \pi /(2 n+3)$, $j=1, \ldots, n$. These define the nodes of case [f]. The nodes of case [d] are the supplements of case [f]. If one drops $\theta_{0}=0$ from case [f], then one obtains the solutions for case [c], and dropping the solution $\theta_{n+1}=\pi$ from the solutions of case [d], one gets the solutions of case [b].

As for the weights of the quadrature formulas, they can be computed in several ways. In [2], the para-orthogonal functions $B_{n}(z, \tau)$ and the para-orthogonal functions of the second kind are used to find the Szegö weights $\lambda_{j}$ as in (2.4). These results are also listed in Table 5.3 if explicit expressions are available. For the cases $[\mathrm{a}-\mathrm{g}]$, there are no explcit expressions for the nodes and hence we do not have explicit expressions for the coefficients of the quadrature formulas. We recall that the Gauss weights are given by $A_{j}=\lambda_{j}$, but that for $j \in\{0, n+1\}$, the weights in the quadrature formula are halved. 


\section{References}

[1] A. Bultheel, P. González-Vera, E. Hendriksen, and O. Njåstad. Orthogonality and quadrature on the unit circle. In C. Brezinski, L. Gori, and A. Ronveaux, editors, Orthogonal Polynomials and their Applications, volume 9 of IMACS Annals on Computing and Applied Mathematics, pages 205-210, Basel, 1991. J.C. Baltzer AG.

[2] L. Daruis, P. González-Vera, and O. Njåstad. Szegő quadrature formulas for certain Jacobi-type weight functions. 1999. Preprint.

[3] P.J. Davis and P. Rabinowitz. Methods of numerical integration. Academic Press, 2nd edition, 1984.

[4] Ya. Geronimus. Polynomials orthogonal on a circle and interval. International Series of Monographs in Pure and Applied Mathematics. Pergamon Press, Oxford, 1960.

[5] U. Grenander and G. Szegö. Toeplitz forms and their applications. University of California Press, Berkley, 1958.

[6] W.B. Jones, O. Njåstad, and W.J. Thron. Moment theory, orthogonal polynomials, quadrature and continued fractions associated with the unit circle. Bull. London Math. Soc., 21:113-152, 1989.

[7] V.J. Krylov. Approximate calculation of integrals. MacMillan, 1962.

[8] G. Szegő. Orthogonal polynomials, volume 33 of Amer. Math. Soc. Colloq. Publ. Amer. Math. Soc., Providence, Rhode Island, 3rd edition, 1967. First edition 1939. 